\title{
РАЗРАБОТКА ИНСТРУМЕНТАРИЯ СТРАТЕГИЧЕСКОГО УПРАВЛЕНИЯ КЛАСТЕРНЫМ РАЗВИТИЕМ РЕГИОНА
}

\author{
(C) 2021 Селентьева Тамара Николаевна \\ ст.преподаватель Высшей Инженерно-Экономической Школы \\ Санкт-Петербургский политехнический университет Петра Великого, Россия, Санкт-Петербург \\ E-mail: selentieva_tn@spbstu.ru
}

Статья раскрывает результаты исследования, посвященного разработке инструментария стратегического управления развитием региональных инновационных кластеров. Актуальность развития инструментария стратегического управления кластерным развитием обусловлена тем, что на сегодняшний день кластерная политика представляет собой одно из важнейших направлений регуляторного воздействия на инновационные процессы региональных социально-экономических систем. Несмотря на наличие большого количества научно-практических публикаций, раскрывающих отдельные вопросы кластеризации региональной экономики, требует уточнения и развития как понятийный аппарат кластерного строительства, так и методический инструментарий управления деятельностью региональных инновационных кластеров. Объектом публикуемого исследования стали механизмы управления, а предметом - процессы инновационной кластерной кооперации на примере кластерных образований Санкт-Петербурга. Теоретико-методологическое значение проведенного исследования заключается в развитии понятийного аппарата кластерного строительства с применением институционального подхода, а также концептуальных основ осуществления стратегического управления кластерным развитием. Практическое значение проведенного исследования заключается в развитии инструментария экспертных оценок применительно к анализу кластерного инновационного взаимодействия в рамках институционального подхода, наряду с использованием метода рандомизированных сводных показателей для выбора объектов государственной поддержки кластерного развития, а также последующего совместного применения сценарного подхода и метода анализа иерархий в рамках формирования стратеги развития региональных инновационных кластеров. В статье последовательно изложены полученные автором результаты теоретико-методического и организационно-практического этапов, раскрыты ключевые предпосылки, определившие характер организации и проведения исследования. Проведенный автором анализ позволил обосновать необходимость применения положений институционального подхода к анализу инновационной кластерной кооперации, а также выработать принципиально новый алгоритм принятия стратегических решений в области управления развитием региональных инновационных кластеров. Научной новизной публикуемого исследования следует считать разработку инструментария оценки трансакционных издержек внутрикластерной инновационной кооперации и последующее применение его результатов в сочетании с другими методами к формированию стратегии развития кластерных структур.

Ключевые слова: кластерная стратегия, региональные инновационные кластеры, экспертные оценки, инновационная кооперация, трансакционные издержки, инфраструктурные связи, институциональный подход, кластерная политика

На сегодняшний день кластерная политика как в отечественной, так и зарубежной практике становится одним из значимых направлений стимулирования инновационной деятельности хозяйствующих субъектов в различных отраслях народного хозяйства, обеспечивая социальноэкономическое развитие региональных систем [1]. В этом отношении вопросы формирования стратегии развития кластеров, выступающих в роли драйверов регионального развития, приобретают особую актуальность, подразумевая решение целого ряда научно-практических задач как на уровне теоретико-методического обоснования, так и требуя проработки организационнопрактической стороны исследования. Данная публикация представляет результаты прове- 
денного автором исследования, целью которого являлась разработка методики формирования стратегии региональных инновационных кластеров (РИК) на основе анализа развития кластерной среды Санкт-Петербурга.

Широкие временные рамки проведения исследования, начатого в 2017 г., позволяют нам сейчас говорить о необходимой для успешного формирования стратегии РИК структуре этапов реализации научно-практических задач. В первую очередь, следует подчеркнуть, что исследование строилось в рамках ставшим на сегодняшний день классическим подхода, требующего первостепенно выработки теоретико-концептуального обоснования предлагаемого методического инструментария. На данном этапе был проведен анализ сущностной природы инновационной деятельности и инноваций, процесса кластерного строительства и его роли в региональном социально-экономическом развитии, кластерной политики в системе государственного регулирования инновационной активности, рассмотрены различные подходы к анализу возникновения кластеров в системе региональной экономики, проанализирован зарубежный опыт развития и поддержки кластеров и проведено сравнение с мерами, принимаемыми в России.

Следует отметить, что российская практика кластерного строительства, получив нормативно-правовое закрепление в Концепции долгосрочного социально-экономического развития в 2008 г. [2], захватывает сравнительно меньший временной период, нежели зарубежный опыт реализации кластерной политики, причиной чему служит множество факторов. Ставшая сегодня хрестоматийной работа М. Портера 1990 г. закрепила превалирующий в современной практике подход к идентификации кластерных структур и легла в основу применяемого в США инструментария анализа кластеров, однако формирование первых практик кластерного строительства в странах Европейского союза принято относить еще к 1980-м гг [3-5]. Анализ отечественной нормативно-правовой базы и документов стратегического планирования социально-экономического развития территорий позволяет утверждать, что идеи кластерного строительства находят широкое отражение в практике принятия управленческих решений как на федеральном, так и на региональном уровне, обусловливая важность рассмотрения вопросов формирования стратегий развития кластеров как первоэлементов кластерной среды [6-8].

Говоря же о различных подходах к рассмотрению возникновения кластерных структур в системе региональной экономики считаем необходимым отметить, что использование положений институциональной теории позволяет наиболее полно проанализировать сущностную природу кластерного образования [9]. В контексте выбранного направления нами были сформированы предложения по развитию терминологического аппарата кластерного строительства, предполагающие уточнение категории кластера через применение понятий инфраструктурных связей и трансакционных издержек, а также учитывающие специфику образования кластерных структур на рынке монополистической конкуренции. Помимо этого, на наш взгляд, применение институционального подхода позволяет раскрыть роль кластерной политики в системе регионального управления. В частности, нами была предложена концепция влияния РИК на формирование региональной конкурентоспособности, описывающая возникновение специфических синергетических эффектов на всех уровнях взаимодействия хозяйствующих субъектов: внутриорганизационном уровне предприятий-участников, в рамках кластера между предприятиями-участниками и на уровне региональной социально-экономической системы.

В рамках теоретико-концептуального обоснования предлагаемого методического инструментария считаем также важным подчеркнуть необходимость определения специфических признаков, идентифицирующих региональные инновационные кластеры. Проведенный анализ нормативно-правовых основ деятельности РИК позволяет нам утверждать, что необходимо как уточнение понятия регионального инновационного кластера, так и разработка базы показателей, способной послужить основой для отнесения результатов деятельности кластерных структур к категории инновационных. Во многом выстраивание четкого нормативного поля должно обеспечить совершенствование механизмов реализации различных видов государственной политики на федеральном и региональном уровне: как кластерной, так и в обла- 
сти промышленности и инноваций. В частности, данное, на взгляд автора, позволит повысить качество принимаемых управленческих решений по государственной поддержке деятельности кластеров. В рамках описываемого исследования автором в качестве ключевого идентифицирующего признака РИК было предложено использование категории инновационной кооперации, которая по своим сущностным характеристикам полностью отвечает описываемым институциональной экономикой отношенческим контрактам. Таким образом, в целях идентификации кластера как инновационного целесообразно первоочередно производить оценку наличия в рамках кластерного образования инновационной кооперации.

Важное значение имеет также и анализ роли кластерной политики в системе государственного управления региональным социальноэкономическим развитием [10]. Отметим, что на сегодняшний день по применяемым инструментарию и механизмам инновационная, промышленная и кластерная политики чрезвычайно близки и выстраивание иерархии подчиненности указанных видов политики представляется достаточно дискуссионным вопросом. Вместе с тем, на взгляд автора, обоснованным является рассмотрение кластерной политики как составной части инновационной и промышленной политик, а также использование категории кластерная промышленная политика, сочетающей в себе базовые принципы как развития промышленности, так и поддержки кластеров, которые, в свою очередь, определяют меры, реализуемые в рамках данного направления государственного регулирования. Призма данного подхода к определению границ регуляторного поля позволила автору также проанализировать институциональные основы кластеризации региональной экономики. Так, опираясь на результаты анализа зарубежной и отечественной практики отдельно хотелось бы отметить специфическую роль особых экономических зон в обеспечении деятельности кластеров как важных элементов, формирующих инфраструктуру кластерного строительства [11]. Резюмируя же результаты сравнительного анализа реализации кластерной политики в России и за рубежом, следует сказать, что используемый сегодня в отечественной практике инструментарий кластерного строительства формировался и совершенствуется на основе передового опыта зарубежных стран, как в сфере методической и организационной поддержки деятельности кластеров, так и при развитии специальных информационных ресурсов, повышающих прозрачность деятельности кластерных структур и выступающих одним из механизмов обеспечения коллаборации в кластерной среде. Однако, по мнению автора, обоснованным для российской практики является дальнейшее развитие нормативного и методического обеспечения деятельности кластерных образований, с учетом, в частности, опыта европейских стран в области реализации оценочных процедур и бенчмаркинга [12]. Следующим этапом, обеспечивающим эффективность организационно-практической части исследования, стал анализ подходов к идентификации кластеров. Общим итогом данного аналитического этапа стал вывод о том, что наиболее распространенным сегодня направлением является выявление кластеров на основе статистики занятости, в разных странах имеющее свои методические особенности. Нами были проанализированы ограничения классического инструментария, а также обоснована необходимость его развития с использованием институционального подхода к анализу кластерных структур, с целью чего также были рассмотрены подходы к оценке трансакционных издержек и межфирменной инновационной кооперации. Наряду с этим, нами были определены уровни принятия решений в области стратегического кластерного развития, как то: уровень региональных органов исполнительной государственной власти, уровень региональных центров кластерного развития, уровень управляющих компаний РИК, а также уровень непосредственного руководства предприятий-участников кластеров. В совокупности описанные выше шаги позволили нам сформировать концептуальную схему проведения исследования.

В рамках организационно-практической части исследования с целью формирования стратегии развития РИК нами было предложено использование двухэтапного анализа трансакционных издержек кластерной инициативы с применением метода экспертных оценок в качестве первого шага моделирования и метода рандомизированных сводных показателей для 
последующего обоснования выбора объектов государственной поддержки кластерного развития, являющей собой, на наш взгляд, один из важнейших элементов стратегического управления развитием кластерных структур. Непосредственный анализ проводился на примере показателей деятельности кластерных образований Санкт-Петербурга с 2017 г.

По мнению автора, применение метода экспертных оценок представляет один из немногочисленных на сегодняшний день способов квантификации информации о трансакционных издержках с учетом их специфической природы и отсутствия практики организации систематического статистического наблюдения в стоимостном разрезе как на уровне кластерных образований, так и на уровне отдельных хозяйствующих субъектов [13]. Для наблюдения нами были выделены издержки поиска альтернатив, заключения контрактов, а также оппортунистического поведения и предложены характеризующие их показатели, в первую очередь, временные. Проведенная оценка развития кластерной среды Санкт-Петербурга позволила составить анкету экспертного опроса. Полученные же в рамках данного этапа результаты позволили провести классификацию исследуемых кластеров по фазам жизненного цикла и определить тенденции кластерного развития [14]. Далее, для иллюстрации процесса выбора объектов государственной поддержки кластерного развития нами был применен метод рандомизированных сводных показателей, представляющий собой эффективный инструментарий, используемый в ситуациях многокритериального выбора [15]. Для ряда исследуемых кластеров это позволило определить наиболее эффективные по показателям кластерной деятельности предприятия-участники. Сами же показатели были сгруппированы по трем категориям: общие характеристики, стоимостные характеристики инновационной деятельности и времен- ные характеристики деятельности предприятия, полученные на первом этапе моделирования в ходе экспертных оценок. Анализ структуры кластерных образований по полученным в итоге данного этапа оценкам, позволил, в свою очередь, обосновать применение сценарного метода при разработке кластерной стратегии.

Заключительным этапом исследования стала разработка концептуальной схемы формирования стратегии развития РИК, предлагаемая методика которой сочетает в себе сценарный подход с применением метода анализа иерархий [16]. На базе проведенного анализа автором были выделены критерии формирования кластерных стратегий, а также разработан базовый перечень мер сценариев формировании стратегии развития РИК. После чего на примере исследуемых кластеров был проиллюстрирован процесс принятия решения по формированию стратегии развития отдельных кластерных структур, а также сформулированы общие рекомендации по совершенствованию кластерной политики в регионе.

Рисунок 1 демонстрирует описанную выше и реализованную автором последовательность этапов научно-практического исследования в рамках разработки инструментария стратегического управления кластерным развитием региона.

Подводя итоги подчеркнем, что полученные в рамках исследования результаты представляют собой практический интерес для широкого круга лиц, принимающих решения на разных уровнях стратегического управления развитием кластеров региона - от субъектов управления на уровне государственной власти до отдельных хозяйствующих субъектов, доказывая необходимость развития и поддержания инфраструктурных связей, возникающих между участниками кластерных образований в ходе инновационной кооперации. 


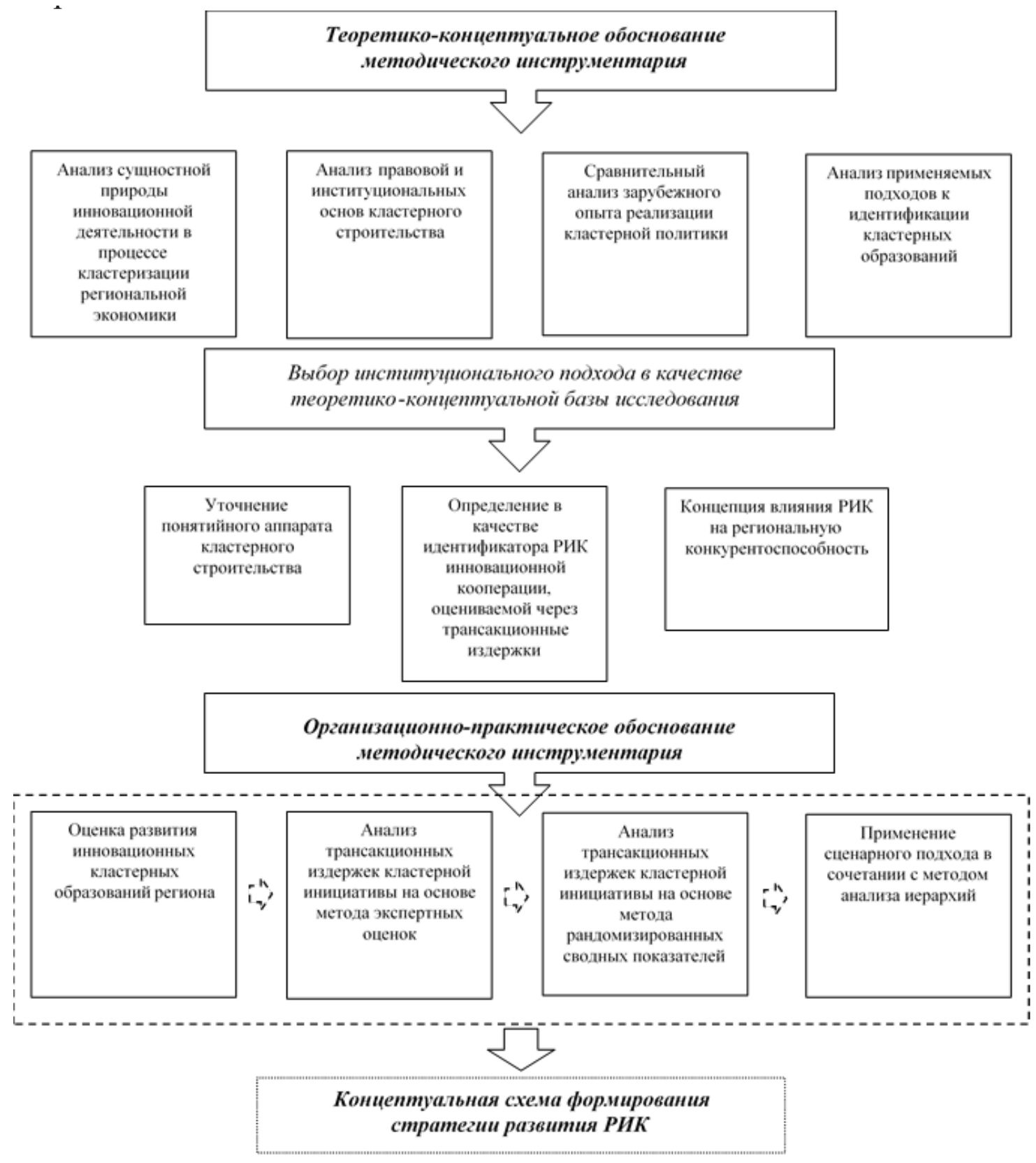

Рисунок 1. Разработка инструментария стратегического управления кластерным развитием региона

\section{Библиографический список}

1. Кудрявцева Т.Ю. Теория, методология и инструментарий кластерной промышленной политики: институциональные и региональные аспекты // Санкт-Петербург: Астерион, 2017-244 с.

2. Распоряжение Правительства Российской Федерации от 17.11.2008 N 1662-р (ред. от 28.09.2018) «О Концепции долгосрочного социально-экономического развития Российской Федерации на период до 2020 года» [Электронный ресурс]. URL: http://www.consultant.ru/document/cons_doc_LAW_82134/28c7f9e359e8af09d724 4d8033c66928fa27e527/ (дата обращения 31.07.2020)

3. Porter M. The economic performance of regions // Regional Studies. - 2003. -, Vol. 37. - P. 549-578.

4. Cluster programmes in Europe and beyond // EOCIC.2019 [Электронный pecypc] URL: https://www. clustercollaboration.eu/news/report-cluster-programmes-europe-and-beyond (дата обращения 30.07.2020)

5. Официальный сайт US Clustermapping [Электронный ресурс] URL: https://www.clustermapping.us/content/ cluster-mapping-methodology (дата обращения 20.12.2018). 
6. Распоряжение Правительства Санкт-Петербурга от 21.05.2019 N 13-рп «О программах развития территориальных кластеров Санкт-Петербурга на 2019-2021 годы» [Электронный ресурс]. URL: http://www.consultant. ru/cons/cgi/online.cgi?req=doc \&base=SPB\&n=212658\#020432097229050217

7. Официальный сайт Центра кластерного развития СПб [Электронный ресурc] URL: https://spbcluster.ru/ (дата обращения: 25.07.2020)

8. Официальный сайт Ассоциации развития кластеров и технопарков России [Электронный pecypc] URL: https://akitrf.ru/ (дата обращения: 25.07.2019).

9. Родионов Д.Г., Кичигин О.Э., Селентьева Т.Н. К вопросу об условиях возникновения кластеров на рынке монополистической конкуренции: институциональный подход // Научно-технические ведомости СанктПетербургского государственного политехнического университета. Экономические науки. 2018. Т. 11 . № 6. C. 54-68.

10. Родионов Д.Г., Кудрявцева Т.Ю. Механизм и принципы формирования кластерной промышленной политики // Инновации. 2018. № 10 (240). с. 81-87.

11. Селентьева Т.Н., Кроливецкий Э. Н. Место и роль особых экономических зон в развитии региональных кластеров // Российский экономический интернет-журнал. 2019. № 1. С. 63.

12. Официальный сайт ESCA [Электронный ресурс]. URL: https://www.cluster-analysis.org/ (дата обращения 31.07.2020)

13. Селентьева Т.Н., Зайцев А.А., Родионов Д.Г. Развитие инструментария экспертных методов для анализа внутрикластерной инновационной кооперации // Бизнес. Образование. Право. 2020. № 4 (53). С. 86-93.

14. Селентьева Т.Н., Зайцев А.А., Карпенко П.А. Оценка тенденций развития внутрикластерной инновационной кооперации в Санкт-Петербурге // Вестник Алтайской академии экономики и права. 2020. № 10-2. С. 167-178.

15. Колодко Д.В. Многокритериальный выбор инвестиционного проекта с помощью метода рандомизированных сводных показателей // Управление экономическими системами: электронный научный журнал.2019. - № 3 (121). [Электронный ресурс] URL: http://uecs.ru/instrumentalnii-metody-ekonomiki/item/54232019-03-16-08-01-31 (дата обращения: 25.07.2020)

16. Зайцев А.А., Сулин М.А. Концептуальные подходы к определению приоритетов государственного регулирования в аграрной сфере региона // Известия Международной академии аграрного образования.-СПб: Издательство МААО, 2015. № 21.-С. 120-125. 